\title{
Patient-Generated Health Data (PGHD) Interoperability: An Integrative Perspective
}

\author{
Rada HUSSEIN ${ }^{\mathrm{a}, 1}$, Rik CRUTZEN ${ }^{\mathrm{b}}$, Johanna GUTENBERG ${ }^{\mathrm{a}, \mathrm{b}}$, Stefan Tino KULNIK ${ }^{\mathrm{a}}$, \\ Mahdi SAREBAN ${ }^{\mathrm{a}, \mathrm{c}}$ and Josef NIEBAUER ${ }^{\mathrm{a}, \mathrm{c}}$ \\ ${ }^{a}$ Ludwig Boltzmann Institute for Digital Health and Prevention, Salzburg, Austria \\ ${ }^{\mathrm{b}}$ CAPHRI Department of Health Promotion, Maastricht University, Maastricht, The \\ Netherlands \\ ${ }^{\mathrm{c}}$ University Institute of Sports Medicine, Prevention and Rehabilitation, \\ Paracelsus Medical University, Salzburg, Austria
}

\begin{abstract}
With advances in Digital Health (DH) tools, it has become much easier to collect, use, and share patient-generated health data (PGHD). This wealth of data could be efficiently used in monitoring and controlling chronic illnesses as well as predicting health outcome. Although integrating PGHD into clinical practice is currently in a promising stage, there are several technical challenges and usage barriers that hinder the full utilization of the PGHD potential in clinical care and research. This paper aims to address PGHD opportunities and challenges while developing the DH-Convener project to integrate PGHD into the Electronic Health Record in Austria (ELGA). Accordingly, it provides an integrative technicalclinical-user approach for developing a fully functional health ecosystem for exchanging integrated data among patients, healthcare providers, and researchers.
\end{abstract}

Keywords. Digital Health, Electronic Health Record, interoperability, PGHD, personalized medicine

\section{Introduction}

Patient-Generated Health Data (PGHD) is defined as "health-related data created and recorded by or from patients outside of the clinical setting to help address a health concern" [1]. Innovations in Digital Health (DH) tools and technologies- such as mobile apps, smartphones, wearables, and connected medical devices- have facilitated PGHD capture, use, and sharing [2]. PGHD usually include biometric data, medication effects, symptoms, and activity levels [3]. Aligning PGHD with clinical treatment pathways has a potential value across the care continuum from prevention, diagnosis, maintenance of health management to treatment intervention [4]. It also improves patients' engagement with care plans [4]. To realize that, data interoperability is a prerequisite for the transformation into personalized digital medicine [5]. Also, all stakeholders' concerns about the PGHD privacy and handling should be addressed [4].

This paper aims to provide an integrative technical-clinical-user perspective on integrating PGHD with the Electronic Health Record (EHR) in Austria (ELGA), through the DH-Convener project. This approach is required to establish PGHD interoperability.

${ }^{1}$ Corresponding Author, Rada Hussein, Ludwig Boltzmann Institute for Digital Health and Prevention, Lindhofstrasse 22, 5020 Salzburg, Austria; E-mail: rada.hussein@dhp.lbg.ac.at. 


\section{Integrating PGHD into health care delivery}

The use of PGHD in clinical practice and research is currently in a promising and expanding stage $[2,3,6]$. There are initiatives in several countries for utilizing PGHD on a national scale, for example:

- In the United States of America, the office of the National Coordinator project for Health IT provided a white paper on PGHD in care delivery informed by two pilot demonstrations (2018) [7].

- In Canada, the TRUSTSPHERE project aims to empower citizens to view, share and manage their own health data through a trustworthy health access platform (2018) [8].

- In Finland, the W2E platform enables citizens to integrate, visualize, and analyze their fitness data (2018) [9].

Nevertheless, most recent studies [10] reported that PGHD integration into EHRs is still in its infancy, as most are still in their pilot phases. There are still no best practices on how to incorporate PGHD into clinical workflows.

\subsection{Opportunities and barriers}

Table 1 summarizes stakeholder perspectives (patients, healthcare providers, and researchers) on PGHD opportunities and barriers for transforming healthcare [2-4].

Table 1. PGHD integration opportunities and barriers

\begin{tabular}{ll}
\hline \multicolumn{1}{c}{ Opportunities } & \multicolumn{1}{c}{ Barriers } \\
\hline - Empowering patients to manage their data & - Patient concerns: data protection, impact on \\
- Engaging patients in healthcare planning & doctor-patient relationships, lack of evidence for \\
- Providing a more holistic view of a patient's & use, and cost reimbursement \\
health and wellness over time & - Provider concerns: insufficient time, lack of \\
- Enabling remote monitoring and timely & expertise to handle information overload, and \\
intervention & lack of knowledge about circumstances of data \\
- Fostering better outcomes and improved & collection and validity, and cost reimbursement \\
workflows & - Technical barriers: lack of PGHD standards, \\
- Providing access to more expansive and diverse & poor data interoperability and accuracy (e.g., \\
datasets to aid in clinical research & structure, completeness, etc.) \\
\hline
\end{tabular}

To address the above concerns, an integrative technical-clinical-user perspective of integrating PGHD with ELGA will be utilized through the DH-Convener project.

\section{The DH-Convener project}

In May 2020, the Austrian BMK ministry organized the Nexus Digital Health Innovation Challenge for shaping the future national health system in Austria. The first author participated with the concept of the DH-Convener for collecting, protecting, and integrating PGHD. The DH-Convener won the Nexus challenge for organizing future health data. This approach's novelty is providing PGHD interoperability and security as a service that matches with the industrial visionary studies on the future of DH transformation by radically interoperable data and open and secure platforms [11]. The convener relies on the person-centered approach to engage the users in developing their own care plan. This will also empower users for directly sharing and controlling their health data for clinical care and research without the need for telehealth platforms. 


\subsection{Technical perspective}

The DH-Convener is an open platform that will support PGHD collection, organization, and interoperability in compliance with the General Data Protection Regulation (GDPR). We aim to create a common data schema for PGHD that can be easily translated into a Clinical Document Architecture (CDA). The PGHD will be secured and stored in the open platform. Therefore, the platform could be connected to ELGA by adding an Integrating the Healthcare Enterprise (IHE) connector and a CDA manager. The first pilot will be implemented at the Salzburg University Hospital (SALK - Affinity Domain). Figure 1 summarizes the three planned modules of the DH-Convener.

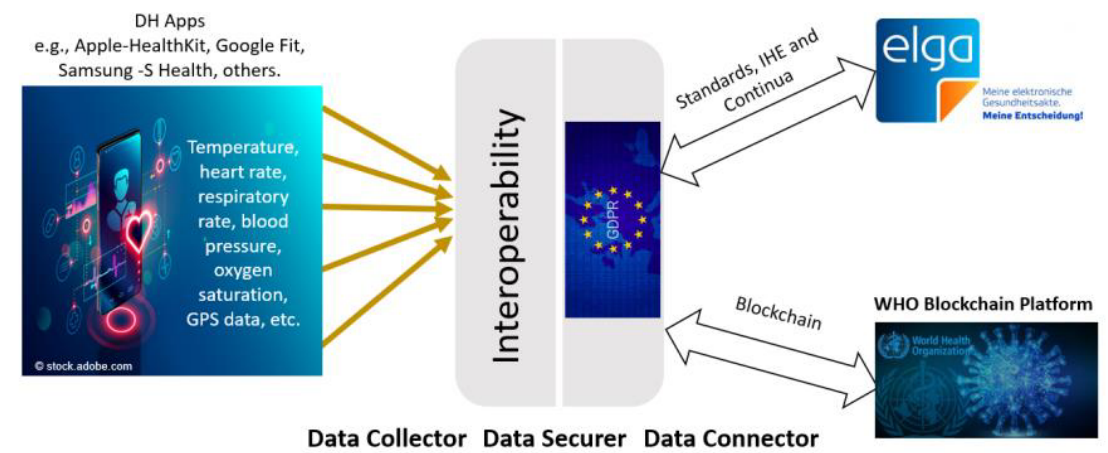

Figure 1. DH-Convener: Open and secure platform for PGHD interoperability and security as a service

- Data collector module: collects and organizes data generated by DH apps, such as temperature, heart rate, respiratory rate, blood pressure, oxygen saturation, etc. This module will also provide PGHD processing and cleaning to improve data quality.

- Data securer module: ensures that PGHD collection and integration conforms to the GDPR compliance requirements in data privacy and security.

- Data connecter module: establishes interoperable health data exchanges between the collected PGHD and ELGA using healthcare standards and interoperability frameworks, namely Health Level 7 (HL7), IHE, and Continua guidelines for personal health systems interoperability. Additionally, this module will adopt the Blockchain technology to facilitate public health data interoperability with the World Health Organization (WHO)'s newly launched Blockchain-based platform MiPasa for monitoring the global pandemics [12].

Ultimately, a big data analytics and visualization module will be integrated.

\subsection{Clinical perspective}

Medical societies highlight treatment recommendations based on the level of evidence, usually derived from meta-analyses or multiple Randomized Controlled Trials (RCTs). However, RCTs report the effect for the collective population included in the trial, which is not necessarily transferable to an individual patient. The advent of personalized medicine demands tailoring treatment to an individual patient. While the concept of personalized medicine started with more attention to genetic data, to date a holistic health 
profiling embraces new fields of PGHD that can provide vast amounts of continuous monitoring data from each individual [13]. Furthermore, data science developed to an extent that allows data processing and medical decision-making support based on artificial intelligence-based analysis of these data sets. Despite this evolving paradigm shift, scientific evidence for improved health-related outcome using the data-driven personalized approach vs. the evidence-based medicine approach is lacking but holds promise to revolutionize how physicians choose, what is best for their patients [6].

To overcome the challenges facing clinicians in using and sharing PGHD, several enabling actions are planned to be taken through the DH-Convener project [3], such as, incorporating prioritized PGHD use cases into clinical workflows, integrating clinicians' perspective during the development of the platform, identifying best practices of PGHD use to strengthen the evidence of PGHD clinical and economic value, and supporting clinicians in patient education on how to capture and share quality PGHD.

\subsection{User perspective}

Any endeavor to establish a PGHD tool will benefit from a thorough understanding and appreciation of the target population and specific user groups, so that their concerns, needs, and expectations may be addressed early in the DH-Convener development. It has been emphasized that this will ultimately make DH tools and technologies not only more user-centered but also GDPR compliant.

With the mandatory application of GDPR in the European Union, citizens became more willing to share their health data [14]. This is also reflected in our recent study of a sample of 25 older adults with cardiac conditions who reside in the Salzburg region of Austria [15]. In-depth exploratory qualitative interviews included questions about data privacy, GDPR, and data sharing, from which several common themes became apparent.

There was a general sense of potential risks of disclosing personal data, for example, on websites or smartphone apps. Additionally, only few participants described the underlying feeling of personal powerlessness and lack of transparency regarding how, where, and by whom data were being used and handled, especially in the digital domain.

Regarding the sharing of personal data, participants described a generally positive attitude but defined two distinct barriers. Firstly, with respect to the type of information that could be shared, the provision of personal health data was mainly considered unproblematic, but a firm line was drawn at data that could reveal socio-economic status by disclosing financial information, income or personal wealth. Secondly, concerning the use of shared information, participants were overall comfortable if this brought personal health benefit (e.g., convenient availability of their own medical history to different doctors) or benefit to science. However, there was clear opposition to the commercial and political use of data.

In this regard, these interview findings stress the utmost importance of building and maintaining trust and transparency between a PGHD service provider and system users, and the necessity to offer users convenient ways to exert control over which types of data are being shared and for which purposes.

Finally, we are catering to a user group with cardiovascular disease who are largely over 55 years of age, and many of whom will have acquired digital literacy towards the end of their working life or after retirement. In the future, we may expect that more open attitudes and expectations concerning data privacy and sharing will be different from digital native generations, who grow up with fitness apps, fitness trackers and smartwatches, and become familiar and comfortable with the continuous data sharing 
and self-monitoring. Those will likely have greater digital health literacy and present differing needs and demands for a PGHD system.

\section{Conclusion}

The DH-Convener project aims to provide PGHD interoperability and security as a service while considering the associated technical-clinical-user aspects. This is based on empowering users to share, own, and control their health and fitness data stored in commercial apps. Integrating PGHD into ELGA (the Austrian electronic health record) has four advantages: 1) widening the adoption of personalized medicine, 2) accelerating the transition toward value-based health systems that rely on quality services, 3) supporting remote health monitoring, and 4) sharing data for public health research.

\section{References}

[1] Accenture. Exploring the potential of patient-generated health data 2018. [cited 2020 Nov 4]. Available from: https://www.accenture.com/_acnmedia/PDF-132/Accenture-Federal-Services-exploring-ThePotential-Of-Patient-Generated-Health-Data.pdf\#zoom $=50$.

[2] Lai AM, Hsueh P-YS, Choi YK, Austin RR. Present and Future Trends in Consumer Health Informatics and Patient-Generated Health Data. Yearb Med Inform. 2017;26(01):152-9.

[3] The Office of National Coordinator for Health Information Technology. Conceptualizing a Data Infrastructure for the Capture, Use, and Sharing of Patient-Generated Health Data in Care Delivery and Research through 2024. Accenture Federal Services; 2018 Jan [cited 2020 Nov 4] p. 67. Available from: https://www.healthit.gov/sites/default/files/onc_pghd_final_white_paper.pdf.

[4] Lavallee DC, Lee JR, Austin E, Bloch R, Lawrence SO, McCall D, et al. mHealth and patient generated health data: stakeholder perspectives on opportunities and barriers for transforming healthcare. Mhealth [Internet]. 2020 Jan 5 [cited 2020 Nov 4];6. Available from: https:/www.ncbi.nlm.nih.gov/pmc/articles/PMC7063266/

[5] Lehne M, Sass J, Essenwanger A, Schepers J, Thun S. Why digital medicine depends on interoperability. npj Digit Med. 2019 Dec;2(1):79.

[6] Demiris G, Iribarren SJ, Sward K, Lee S, Yang R. Patient generated health data use in clinical practice: A systematic review. Nursing Outlook. 2019 Jul;67(4):311-30.

[7] Patient-Generated Health Data | HealthIT.gov [Internet]. [cited 2020 Nov 4]. Available from: https://www.healthit.gov/topic/scientific-initiatives/patient-generated-health-data

[8] TRUSTSPHERE | Digital Technology Supercluster [Internet]. [cited 2020 Nov 4]. Available from: https://www.digitalsupercluster.ca/programs/precision-health/trustsphere/

[9] W2E | Wellness data integration made easy [Internet]. [cited 2020 Nov 4]. Available from: https://w2e.fi/frontpage/

[10] Tiase VL, Hull W, McFarland MM, Sward KA, Fiol GD, Staes C, et al. Patient-generated health data and electronic health record integration: protocol for a scoping review. BMJ Open. 2019 Dec 1;9(12):e033073. Forces of Change - The future of health | Life Science and Health | Deloitte [Internet].

[11] Forces of Change - The future of health | Life Science and Health | Deloitte [Internet]. Deloitte Insights. [cited 2020 Nov 25]. Available from: https://www2.deloitte.com/us/en/insights/industry/healthcare/forces-of-change-health-care.html

[12] What is MiPasa? [Internet]. [cited 2020 Nov 6]. Available from: https://mipasa.org/about/

[13] Hayes, D. F., Markus, H. S., Leslie, R. D., \& Topol, E. J. (2014). Personalized medicine: risk prediction, targeted therapies and mobile health technology. BMC medicine, 12, 37. https://doi.org/10.1186/17417015-12-37

[14] Karampela, M., Ouhbi, S., \& Isomursu, M., 2019. Exploring users' willingness to share their health and personal data under the prism of the new GDPR: implications in healthcare. In 2019 41st Annual International Conference of the Ieee Engineering in Medicine and Biology Society (embc) (pp. 65096512). IEEE.

[15] Gutenberg, J., ulnik, S. T., Hussein, R., Stütz, T., Niebauer, J., \& Crutzen, R. (2020). Physical Activity in Cardiac Rehabilitation: Towards Citizen-Centered Digital Evidence-Based Interventions. Studies in Health Technology and Informatics, 275, 228-229. 